\title{
Contradicciones de la Amazonía en el Brasil: Una nueva perspectiva
}

\author{
Contradictions of the Amazon in Brazil: A new outlook
}

\author{
Creucí María Caetano* (iD Diego Geraldo Caetano Nunes
}

\section{Acceso Abierto}

\section{*Correspondencia:}

cmcaetano@unir.br

Universidade Federal de Rondônia

(UNIR), campus Presidente Médici.

R. da Paz, 4376 - Lino Alves Teixeira,

Pres. Médici - RO, 76916-000, Brasil.

Recibido: 14-03-2021

Aceptado para publicación:

26-05-2021

Publicado en línea: 09-09-2021

Palabras clave:

Amazonía brasileña;

cambio climático;

deforestación:

especies nativas;

megabiodiversidad;

monocultivo;

suelo tropical.

Key words:

Brazilian amazon;

climate change;

deforestation;

native species;

megabiodiversity;

monoculture;

tropical soil.

\section{Citación:}

Caetano CM, Caetano Nunes DG. Contradicciones de la Amazonía en

el Brasil: Una nueva perspectiva. Magna Scientia UCEVA 2021; 1 : 68-77. https://doi.org/10.54502/ msuceva.v1n1a10.

\section{Resumen}

La foresta amazónica se destaca por ser uno de los más importantes componentes de la biosfera, fundamental para afrontar los cambios climáticos, debido a que se caracteriza por albergar una megadiversidad biológica, étnica y cultural, que sufre en la actualidad una intensa presión. Tal presión, puede ser caracterizada como sigue: $i$ ) la deforestación creciente, con la tala ilegal de árboles nativas y las quemas, con consecuentes cambios en el uso del suelo tropical; por lo general, indebidamente manejado con implementación de monocultivos y técnicas inadecuadas para estos tipos de suelos; ii) la invasión a tierras indígenas y áreas de unidades de conservación, poniendo en riesgo el patrimonio sociocultural y la sociobiodiversidad; además, compromete también el patrimonio genético, del cual, un número expresivo de especies no son todavía conocidos y que podrían contrarrestar adversidades relacionadas con el cambio climático. Bajo esta perspectiva, la presente reflexión busca traer a la luz, algunos puntos vinculados con las diferentes problemáticas que tienen como escenario la Amazonia brasileña. Más que reflexionar, se trata de hacer un llamado hacia una nueva mirada de esta región amazónica, en caso contrario, se puede estar asistiendo a una masacre de pueblos originarios, de conocimientos tradicionales y de recursos genéticos.

\section{Abstract}

The Amazon forest stands out for being one of the most important components of the biosphere, fundamental to face climatic changes, due is characterized by hosting a megabiological, ethnic and cultural diversity, which is currently under intense pressure. Such pressure can be characterized as follows: $i$ ) growing deforestation, with illegal logging of native trees and burning, with consequent changes in the use of tropical soil; generally, improperly managed with monocultures implementation and unsuitable techniques for these types of soils; ii) the invasion of indigenous lands and areas of conservation units, putting sociocultural heritage and sociobiodiversity at risk; in addition, also compromises the genetic heritage, of which a significant number of species are not yet known and which could counteract adversities related to climate change. From this perspective, this reflection seeks to bring to light, some points related to the different problems that are set in the brazilian Amazon. Rather than reflect, is about calling for a new look at this Amazon region, otherwise, can be witnessing a massacre of native peoples, traditional knowledge and plant genetic resources. 


\section{Introducción}

En las últimas tres (3) décadas, la Amazonía brasileña ha sufrido un proceso impactante [1], cambios en el paisaje natural, deforestación, quemas indiscriminadas y criminosas [2]; la explotación de madera nativa y establecimiento de minería ilegal, especialmente, en territorios indígenas y en áreas de preservación permanente (APPs) [3]. Si no se dan condiciones para que ocurra el fenómeno de evapotranspiración de los árboles [4], sumado a la evaporación del agua proveniente del Océano Atlántico, la cual es transportada desde la franja ecuatorial por los vientos alisios en sentido leste-oeste [5], tampoco se da la formación de los ríos voladores o atmosféricos, científicamente conocidos como "Jatos de Bajos Niveles de la América del Sur', los cuales influencian el clima no solo en la región amazónica, sino más allá, en el cono sur [6]; y en otros biomas, como el pantanal Matogrossense que, al igual que la Amazonía, sufrió duramente el año 2020 [7], una drástica sequía y quemas durante meses, además de soportar una reducción significativa de su biodiversidad, trayendo consigo, pérdidas irreparables en este reconocido ecosistema [8].

Cambios en el paisaje cultural y en la socio-biodiversidad de la Amazonía brasileña, como la ocurrida en los pueblos originarios de distintas etnias en extrema vulnerabilidad, aún más, cuando la Covid-19, los asola con mayor crueldad; comunidades tradicionales como los Quilombolas, que son remanentes de los Quilombos o Palenques [9] y los Ribeirinhos, poblaciones que viven a las orillas de ríos o de Igarapés, practicando la pesca artesanal y una agricultura de pan coger; ambas, ignoradas por los Entes gubernamentales [10] [11]; y aún más, poblaciones locales que practican la agricultura familiar, en su mayoría agroecológicas, y las que subsisten a partir de la extracción sustentable de productos forestales no maderables (PFNM) como el caucho [Hevea brasiliensis (Willd. ex A.Juss.) Müll. Arg.]; fibras diversas; frutos y semillas como el guaraná (Paullinia cupana Kunth), cacao (Theobroma cacao L.), copuazú [Theobroma grandiflorum (Willd. ex Spreng.) K.Schum.], açaí (Euterpe oleracea Mart.), el chontaduro (Bactris gasipaes Kunth.), la nuez de Brasil (Bertholletia excelsa Bonpl.), entre otras especies nativas igualmente desasistidas.
En este mismo sentido, toman partida los conflictos socioambientales como el uso del suelo y posesión de tierras, la ganadería bovina extensiva. Cabe mencionar que en el Estado de Rondônia-Brasil, existía en el año 2020, según datos oficiales del Instituto Brasileiro de Geografia e Estatística (IBGE), 7.9 cabezas de ganado por habitante [12]; la introducción de monocultivos, especialmente el cultivo de la soya (Glycine max (L.) Merr.), sin respetar las secuencias de suelos tan peculiares de la Amazonía ni los ciclos del agua y de nutrientes [13].

Con el ánimo de elevar un recordatorio, vale la pena considerar que la Amazonía no es solo forestas; está constituida por otras fitofisionomías, como el cerrado (o sabana) con sus diversas fisionomías, y los campos generales o Lavrados; los cuales, desafortunadamente, son en la actualidad, tratadas de la misma manera por sus explotadores [14]. Todo lo anterior, conforma un escenario desolador, al menos en el territorio brasileño, para ésta que todavía es considerada la mayor foresta tropical de la Tierra, la selva amazónica; reconocida por tener el área más grande de selva tropical continua en el planeta, por su gran riqueza en biodiversidad, y por la cultura de sus pueblos indígenas, bien señalada por Davis en su épica obra El Río [15], “...los curanderos nativos como los Aucas, son pragmáticos científicos activos, cuyo trabajo refleja las necesidades sociales y su laboratorio, resulta ser la selva"[15].

Con esta inmensa lista de cambios, está comprometido el concepto generado por el Ministerio de Medio Ambiente de Brasil, según el cual, "el bioma Amazonía es un mundo verde y vasto de aguas y bosques, donde las copas de los enormes árboles ocultan la luz húmeda, la reproducción y la muerte de más de un tercio de las especies de la Tierra" [16].

Amazonia, también el bioma más grande de Brasil, ocupando un área de 4.196 .943 millones de $\mathrm{km}^{2}$ o 419.694.300 ha [17], más de $40 \%$ del territorio brasileño, con 2500 especies de árboles (o una tercera parte de toda la madera tropical en el mundo) y 30000 especies de plantas (de 100000 en América del Sur), se encuentra en riesgo inminente [18]. Una realidad que no es tan diferente en la mayoría de los demás países que comparten con Brasil, la foresta amazónica en Sur América, Bolivia, Colombia, Ecuador, Guyana, Guyana Francesa, Perú, Surinam y Venezuela [19]. 
Por lo tanto, la presente, es una reflexión sobre ésta, que es un importante componente de la biosfera, la foresta amazónica, supuestamente defendida por ambientalistas, organizaciones no gubernamentales, misioneros evangélicos y gobiernos de países desarrollados, por ser ¿el (pseudo) pulmón del mundo?; ¿fuente inagotable de minerales y piedras preciosas?; ¿más grande reserva mundial de maderas?; ¿su gran biodiversidad?; ¿Y quiénes se preocupan con sus pueblos originarios, su socio biodiversidad, o, la geografía humana?; el propio gobierno brasileño (2019-2022), va en contravía cuando se trata de la Amazonía. Como bien puntuó Primavesi "el problema ecológico no tiene solución mientras exista el problema social" [20].

\section{La Amazonía brasileña}

De los Estados que componen la llamada Amazonía Legal en Brasil, se reconocen el Acre,Amapá,Amazonas, Maranhão, Mato Grosso, Pará, Roraima, Rondônia y Tocantins que, en conjunto, atingen una superficie aproximada de $5.015 .067,749 \mathrm{~km}^{2}(501.506 .774 .900$ ha), correspondiente a cerca de $58.9 \%$ del territorio brasileiro [21]. Siete de esos Estados, corresponden a la Región Norte de Brasil, mientras que Maranhão, hace parte de la Región Nordeste, Mato Grosso, de la Región Centro-Oeste del Brasil [22].

La cuenca del Amazonas es la más grande del mundo, cubriendo aproximadamente $6.000 .000 \mathrm{~km}^{2}$ (600.000.000 ha), con 1.100 afluentes. Su principal río, el Amazonas, corta la región para desembocar en el Océano Atlántico, vertiendo, aproximadamente, 175.000.000 de litros de agua por segundo [23]. Según los cálculos, la región es la mayor reserva de maderas tropicales en el mundo, sus recursos naturales que, además de madera, incluyen las enormes reservas de caucho, las nueces, el pescado y los minerales; por ejemplo, representan una fuente abundante de riqueza natural [24].

La Amazonía brasileña es también el hogar de una riqueza cultural, incluyendo el conocimiento tradicional asociado al patrimonio genético y la forma de utilizar los recursos naturales sin agotarlos o destruir el hábitat natural [25]. La Amazonía pudo tener una población de 4 a 5 millones de habitantes a la llegada de los europeos en 1492; hoy, no pasan de 900.000 habitantes [26].
Estos pueblos cultivaron y/o domesticaron por lo menos, 138 especies vegetales, muchas de las cuales, eran artefactos humanos, es decir; se encontraban en un estadio avanzado de domesticación. Consecuentemente, existió una relación entre la reducción de las poblaciones indígenas de la Amazonía y la pérdida de sus recursos genéticos después del contacto. Tal relación fue influenciada por el grado de domesticación del cultivo, su historia de vida, el grado de domesticación del paisaje en la que era cultivado, el número de sociedades indígenas que lo utilizaron y su importancia para estas sociedades.

La historia de la ocupación y colonización de la Amazonía Brasileña ha tenido diferentes fases, siendo las de mayor expresión los dos ciclos del caucho [Hevea brasiliensis (Willd. ex A.Juss.) Müll.Arg.] (18791912;1942-1945), los asentamientos de reforma agraria incentivados por el gobierno brasileño en las décadas de 1960 - 1970, y más recientemente, la presión ganadera, las actividades de madereros y el cultivo de la soya (Glycine $\max$ (L.) Merr.), que han ejercido mayor presión sobre esta región [27].

El proceso de colonización tuvo como una de las principales razones, los incentivos del gobierno federal militar (1964 - 1985), con el argumento de tratarse de una cuestión de seguridad nacional, estimulando asentamientos humanos en áreas antes despobladas [28], con el lema "integrar para no entregar", para asegurarse de que esta porción de la Amazonía, permaneciera brasileña.

El slogan "tierras sin hombres para hombres sin tierra", olvidando completamente de los pueblos originarios amazónicos, lo que enmarcó el Plan de Integración Nacional (PNI) del gobierno federal en los años 70's, responsable por el intenso flujo migratorio de regiones castigadas por la sequía en el Nordeste y por la mecanización agrícola en el Sur, rumbo a la Amazonía [29]; ese fenómeno de migración interno, generó áreas deforestadas, a partir de aberturas de carreteras como la BR-364, la BR-319 y la BR-230.

La BR-364, se inicia en la ciudad de Cordeirópolis (Estado de São Paulo), cruza por Minas Gerais y Goiás, ligando las capitales Cuiabá (Mato Grosso), Porto Velho (Rondônia) y Rio Branco (Acre) y finalizando en la frontera con el Perú, por el municipio de Cruzeiro do Sul, Estado de Acre [21], permitiendo el acceso a nuevos territorios de 
Brasil, desarrollándose poblados, algunos de los cuales son importantes municipios actualmente, construidos a las orillas de la carretera. Grandes áreas de bosque fueron ocupadas por actividades agropecuarias, especialmente pasturas que son, en su mayoría, mal manejadas o de baja producción por unidad de animales [30].

Aunque sean $885 \mathrm{~km}$ en toda su extensión, aproximadamente $450 \mathrm{~km}$ están pavimentados en la BR-319, inaugurada en 1976 [29]; la única ligación por tierra de la capital del Estado del Amazonas, Manaus, a Porto Velho, en Rondônia y de ahí, para el resto de Brasil. Símbolo del fracaso del proyecto de integración conducido por la dictadura militar, la carretera corta uno de los bloques más preservados de la foresta amazónica [31].

La dificultad de locomoción desacelera la llegada del "arco de deforestación" a la región, pero la actual reconstrucción de la vía, ha conllevado actividades ilegales, a la exposición de pueblos indígenas y comunidades tradicionales; el avance de la frontera agrícola, y daños socio-ambientales irreparables [32] (figura 1).

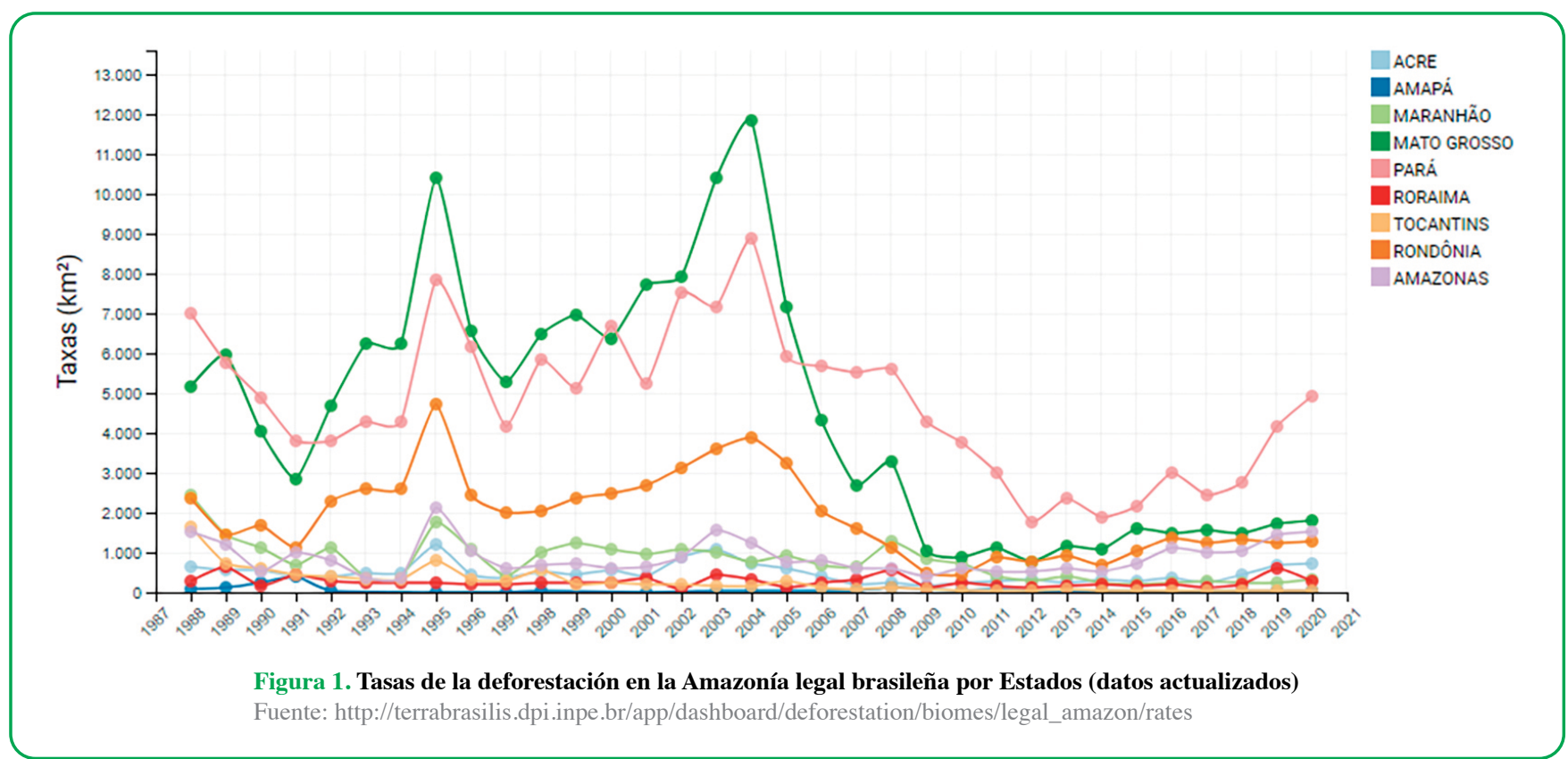

La zona de influencia de la BR-319, comprende 270000 $\mathrm{km}^{2}$ entre los ríos Purus y Madeira, área correspondiente al 5.4\% del territorio de la Amazonía legal [21]. Mientras la porción sur de tierra entre los ríos, es una de las más degradadas de Brasil; la parte norte, registra baja presencia humana, caso del Medio Purus, donde vive el pueblo Apurinã, que denuncia el prenuncio de más un genocidio [29].

El área abriga aún 25 unidades de conservación (UCs) federales y estatales en elAmazonasy en especial,Rondônia. Juntas, forman un extenso corredor de biodiversidad, preservando especies en extinción y endémicas. A pesar de las denuncias y disputas en las instituciones del poder judicial brasileño, los últimos resultados, se han mostrado favorables al desarrollo económico [33].
Al respecto, Éder Carvalho Assunção, párroco de la prelacía de Lábrea, comunidad Belo Monte, enfatiza que, a lo largo de las orillas de carreteras secundarias, "grileiros" o ladrones de tierras, hacen un falso catastro rural, diciendo ser funcionarios del Instituto Nacional de Colonização e Reforma Agrária (INCRA), distribuyendo documentos ilegales. La persona lo recibe como un título de propiedad, pero son tierras de la Unión. La carretera [29], un aparente desarrollo por el hecho de auxiliar la locomoción entre las capitales,se convierte en un camuflaje, debido a que trae consigo, la destrucción de hábitats y, más drástico, de etnias y culturas [34].

Desafortunadamente, así como el pueblo Juma, otras etnias diversas, entre ellas, los Paiter Surui, Puruborá, Tuyuka, Karipuna (menos de 30 personas en su aldea, la 
que más sufrió con las quemas en el 2019) [35], han sido victimizadas por la Covid-19, perdiendo sus mayores médicos tradicionales y conocedores de un inmenso saber ancestral, incluyendo su lengua, rituales, fiestas tradicionales; una pérdida irreparable, exponiéndolos a la vulnerabilidad cultural y social [36] (figura 2).

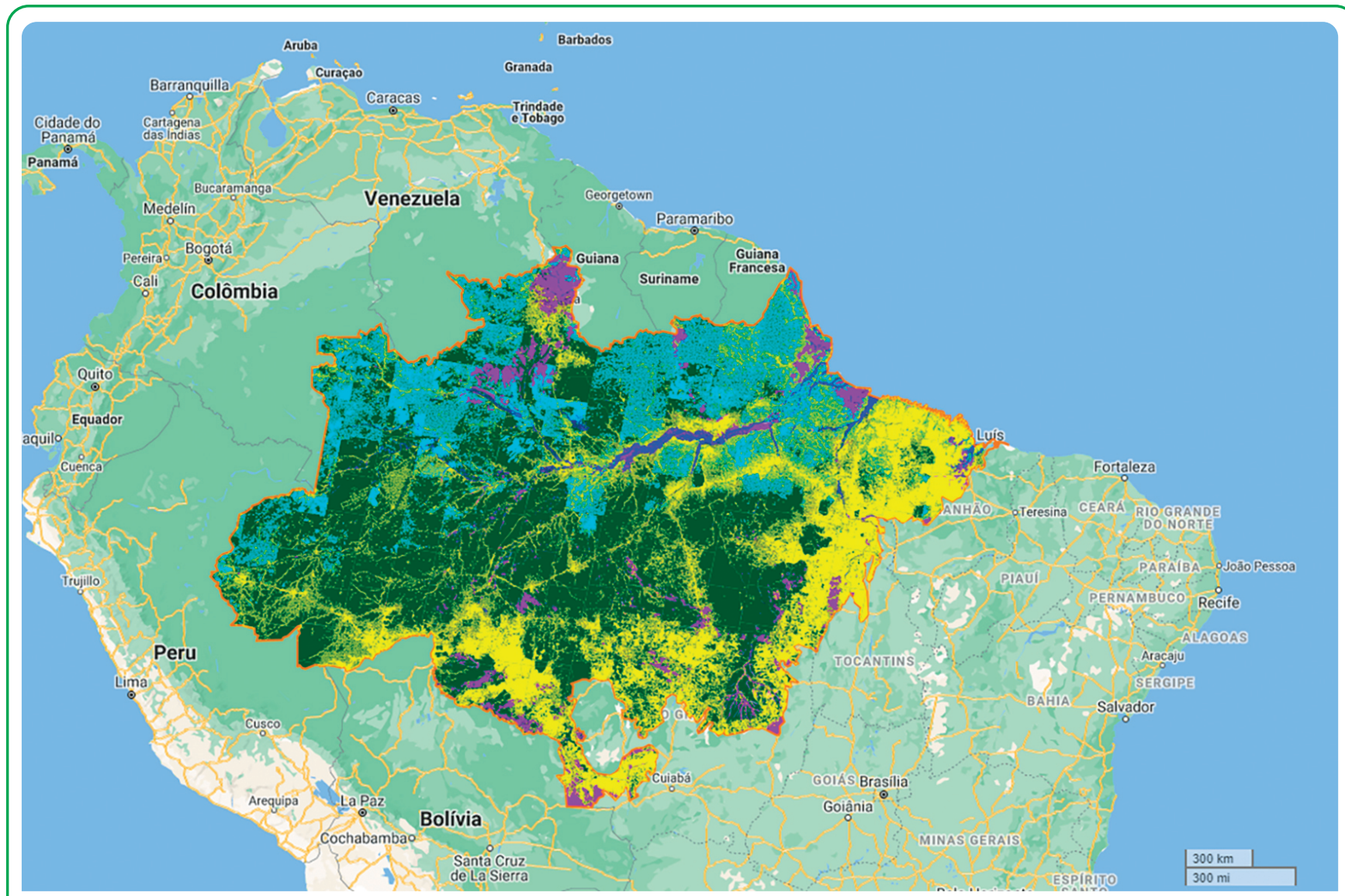

Figura 2. Máscara de la deforestación. Deforestación (amarillo); foresta amazónica (morado); hidrografía (azul). Años 1988-2007 en el bioma Amazonía de la Amazonía brasileña.

Fuente: http://terrabrasilis.dpi.inpe.br/app/map/deforestation?hl=pt-br

Eliézer Puruborá (92 años), uno de los últimos indígenas hablantes de la lengua Puruborá (pueblo originario amazónico, hoy con solo 220 personas), también murió debido a la Covid-19 en el 2020 [37]; amenazadas desde la llegada de los europeos, solo 180-200 de las 1500 lenguas indígenas son habladas actualmente en el Brasil, la mayoría por menos de 1000 personas [38].

La BR-230, el brazo oriental de la llamada Transamazónica, es uno de los legados más contradictorios de la dictadura militar brasileña [34]. Inaugurada en 1972, con $4223 \mathrm{~km}$ de extensión, atraviesa siete Estados, desde el puerto de Cabedelo (costa de Paraíba) hasta Lábrea (sur de Amazonas). Sin embargo, más de un tercio de la BR-230 está en tierras del Pará, los 1840 km iniciales, desde Brejo Grande del Araguaia (Tocantins), está casi toda pavimentada [29].

En la división Tocantins-Pará, el puente sobre el río Araguaia, es el límite para el polvo en el verano o el lodo en el invierno. La degradación de la foresta Amazónica, ocurre con violaciones de derechos, promesas no cumplidas, entre otros aspectos. La historia de la Transamazónica en Pará, se confunde con la de hombres y mujeres que construyeron sus vidas a orillas de la carretera o en las comunidades vecinas [34].

El INPE (Instituto Nacional de Pesquisas Espaciais), a través de su programa de sensores remotos para la Amazonía [39], realiza el monitoreo de la región, 
tomando como base las acciones de investigación y desarrollo direccionadas a la evaluación del impacto ambiental aplicable a los programas energéticos y minerales [40], la expansión de la frontera agrícola, la dinámica actual de uso del suelo, los procesos de erosión y transporte de sedimentos a los ecosistemas acuáticos, entre otros aspectos [41]. A su vez, su proyecto PRODES-Amazônia [42], objetiva detectar y cuantificar la alteración de la cobertura forestal de la Amazonía Legal, mostrando la real situación de la deforestación [40] (figura 2).

El grado de deforestación en la Amazonía en Brasil, se ha convertido en una amenaza de tal dimensión para el equilibrio del ecosistema y su megabiodiversidad, problemática ampliamente registrada en varios estudios [1-3,19,26]. Según el PRODES-Amazônia [42], los datos de intervención antrópica (deforestación seguida por quema) abarcaron en el 2019, para el Estado de Pará- Brasil, $271862.57 \mathrm{~km}^{2}$, Mato Grosso 218741.90 $\mathrm{km}^{2}$, Maranhão $106510.04 \mathrm{~km}^{2}$, Rondônia 94767.13 $\mathrm{km}^{2}$, Amazonas $41895.57 \mathrm{~km}^{2}$, Tocantins 30663.63 $\mathrm{km}^{2}$, Acre $24033.01 \mathrm{~km}^{2}$, Roraima $11311.12 \mathrm{~km}^{2} \mathrm{y}$ Amapá $3112.88 \mathrm{~km}^{2}$ [42]. En el 2020, las pérdidas fueron todavía más drásticas.

De acuerdo con Primavesi [20], cada año, son desertificados más de 10.000 .000 ha de tierras agrícolas en el planeta, en parte por la salinización debida a riegos sin mayores cuidados, y en parte, por las quemas frecuentes. El gran perjuicio causado por el fuego es el no regreso de la materia orgánica y la exposición del suelo desnudo, quemado, al impacto de las lluvias y al sol caliente. La biota del suelo muere de hambre, debido a que la materia orgánica es su alimento y no se la puede cambiar por ceniza, exclusivamente mineral [20]. En términos legales, la Ley más representativa para esta región es la Ley 12651 del 25 de mayo de 2012, conocida como la Ley de Protección de la Vegetación Nativa [43] (figura 3); entre los puntos que se abordan en esta Ley están la definición, función, uso y protección de las áreas de preservación permanente (APP) y de reserva legal (ARL).

Se entiende por Área de Preservación Permanente (APP), un área protegida cubierta o no cubierta por área de vegetación nativa, con la función ambiental de preservar los recursos hídricos, el paisaje, la estabilidad geológica y la diversidad biológica, facilitar el flujo genético de fauna y flora, la protección del suelo y garantizar el bienestar de las poblaciones humanas (figura 3) [44]. A su vez, la Reserva Legal (ARL), es el área que se encuentra dentro de una propiedad rural, con la función de garantizar la utilización económica de los recursos naturales de la propiedad rural de manera sostenible [44]; ayuda a la conservación y rehabilitación de procesos ecológicos y a promover la conservación de la biodiversidad, así como refugio y protección de la fauna y flora nativas [21].

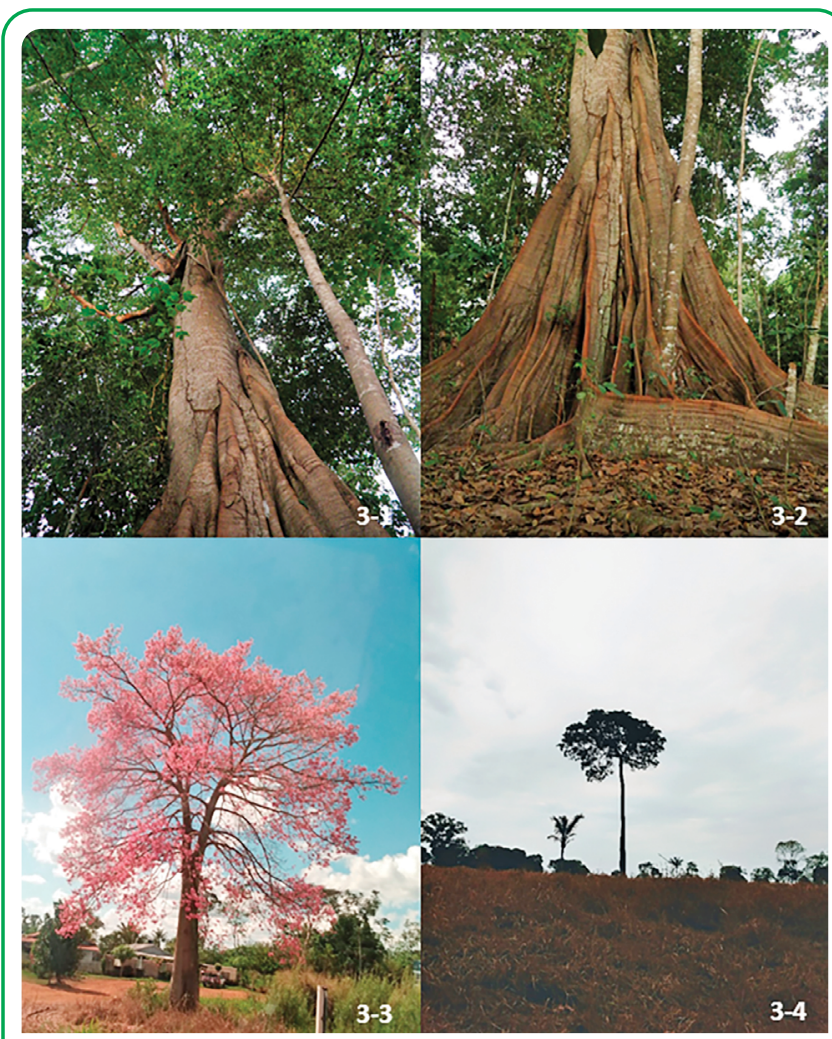

Figura 3. La megabiodiversidad de la foresta amazónica. Representada por especies como la "sumaúma" (Ceiba pentandra (L.) Gaertn.) (31); y el ceibo o "paineira" (Ceiba speciosa (A.St.-Hil.) Ravenna); (32), ambas de la familia Bombacaceae y Malvaceae. La nuez del Brasil, "Castanheira" (Bertholletia excelsa Bonpl.); (3-3; 3-4), una especie cuya tala está prohibida; se destaca en un paisaje con pastura. Sin embargo, así aislada, se encuentra vulnerable a la caída por rayos, al fuego, a la ausencia de polinización por la distancia a otros especímenes, o a la no dispersión de las semillas por su agente dispersor, el mamífero roedor Dasyprocta aguti o "cutia".

Sin embargo, la conservación y la recuperación de ecosistemas nativos en propiedades rurales privadas (ASP) son fundamentales, una vez que estas ocupan cerca de $80 \%$ del territorio brasileño [43]. Por lo tanto, a la situación actual, se hace preponderadamente necesario el cambio en la comprensión del papel de las zonas 
rurales en la protección y conservación de los recursos naturales. El agricultor se convirtió en protagonista en la gestión de sistemas complejos y diversificados, integrados e interdependientes. La conciliación de la eficiencia económica, la responsabilidad social y la protección del patrimonio natural, proporcionando al mismo tiempo, los servicios de los ecosistemas a la sociedad, se han convertido en puntos estratégicos y fundamentales para el productor rural amazónico continuar sus actividades, no de explotación y si desde una visión sustentable.

Con relación a los pueblos originarios, no solo amazónicos como en todo el país, un buen número de observadores nacionales e internacionales concluye que el (des)gobierno federal actual (2019-2022), viene promocionando el más grande ataque a la dignidad, a la cultura y a los derechos indígenas de las últimas décadas, además de la visión despreciativa y pre-conceptuosa sobre pueblos originarios. El anterior director de la FUNAI (Fundação Nacional do Índio), Sydney Possuelo, defensor de los derechos de pueblos indígenas, expresó que, en 42 años de trabajo en la Amazonía, este es el más peligroso que ha presenciado [45].

La posesión de sus tierras, es la gran reivindicación de los pueblos indígenas brasileños en la actualidad, debido a que la tierra es la raíz de valores fundamentales para sus culturas. Sin embargo, cerca del $90 \%$ de todos los procesos demarcatorios están en juzgados, donde las deliberaciones se arrastran por décadas y, las tierras previamente demarcadas, son con frecuencia, invadidas o espoliadas con el beneplácito del gobierno y de la sociedad [46].

Muchos indígenas ya viven en ciudades, forzados a la migración por la expulsión de sus tierras, o por las difíciles condiciones de subsistencia en reservas pequeñas y exhaustas, o buscando las ciudades espontáneamente, para mayor confort, reconocimiento, tratamiento de salud, educación u otros motivos, pero van ilusionados muchas veces encontrando condiciones más duras, viviendo en favelas (cortijos o barrios deprimidos), con dificultades para preservar las tradiciones, o, por fuerza de un contexto desfavorable, abandonándolas. Su visibilidad, ha permitido sensibilizar la población en general, pero permanecen entre los grupos urbanos más desamparados; al margen de la sociedad al igual que otras minorías desasistidas.
Es cada vez más creciente el número de denuncias, sin que se tomen medidas efectivas para resolver esta problemática [47].

El gobierno no cumple con su actual y propia carta magna, la Constitución de la República Federativa do Brasil, de 1988, no está protegiendo actualmente a su pueblo y a su ambiente; tampoco su compromiso con el desarrollo sustentable global. Se han cuestionado los datos de la deforestación provenientes del mismo INPE, el cual muestra el avance del "arco de la deforestación". Y también, se ha cuestionado la más grande aprensión jamás ocurrida en la historia de la Amazonía brasileña, la madera nativa talada ilegalmente, denunciada por la Policía Federal a finales de diciembre de 2020, en la Operación Handroanthus GLO, en la que se estima que cerca de 65 mil árboles nativos fueron exterminados [48].

Al denunciar el Ministro del Medio Ambiente y un senador por ser cómplice de los madereros, y al jefe del IBAMA por omisión, el delegado superintendente del Amazonas de la PF perdió su puesto, en mediados de abril de 2021 [48], en más una medida arbitraria, por parte del gobierno o sus subordinados, terminaron sumisos a su ideología perversa. Desafortunadamente, una ideología política adversa a la conservación de la diversidad biológica, de la sociobiodiversidad, del ecosistema amazónico, un grande descaso del gobierno con relación a su gente - su discurso inconsistente durante la Cumbre del Clima de 22 y 23 de abril de 2021 intentó demostrar al contrario, Brasil, uno de los países que más presenta pérdidas relacionadas con la Covid-19 y la crisis económica paralela; no puede contar con decisiones gubernamentales serias y asertivas. Por todo lo anterior, la gran foresta amazónica merece una nueva mirada, un nuevo entendimiento sobre la mejor forma de desarrollo a ser aplicada, tanto a nivel interno, como internacional.

\section{Consentimiento de publicación}

Los autores leyeron y aprobaron el manuscrito final.

\section{Conflicto de intereses}

Los autores declaran no tener conflictos de interés. Este documento solo refleja sus puntos de vista y no los de las instituciones a las que cada autor pertenece. 


\section{Perfil de autoría}

\section{Creucí Maria Caetano}

Graduada en Ciencias Biológicas (1981), con Maestría en Biología Celular (1995) y $\mathrm{PhD}$ en Ciencias Biológicas (2001) de la Universidade Estadual de Maringá (UEM), Paraná. Pedagoga (UEM; 1994), con un perfeccionamiento en Ciencia y Ciudadanía- Ethos Ciencia de la Faculdade Estadual de Campo Mourao, Paraná (1996), Especialización en Control y Gestión

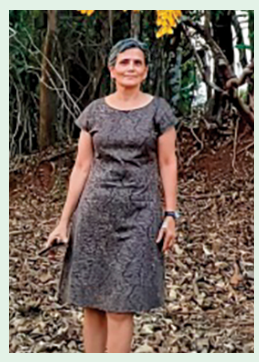
Ambiental (UEM, 1999) y Curso Internacional de Posgrado en Patrimonio Cultural, Turismo Sustentable y Paisaje Cultural (Fondo Verde; 2017). Sus áreas de actuación son la Citogenética Vegetal, la Etnobotánica aplicada, la Conservación y Utilización de Recursos Fitogenéticos (RFG). Ha estado vinculada con el Instituto Internacional de Recursos Fitogenéticos (IPGRI) (actual Bioversity International), la Universidad Nacional de Colombia y la Fundacao Universidade Federal de Rondônia, na Amazônia Ocidental Brasileira, campus Presidente Médici, Departamento de Engenharia de Pesca.

\section{Diego Geraldo Caetano Nunes}

Biólogo de la Faculdade de Ciências Biomédicas de Cacoal (2008), Maestría en Ciencias Biológicas de la Universidad Nacional de Colombia campus Palmira (2012) y PhD en Agroecología, Universidad Nacional de Colombia campus Palmira (2017). Tiene experiencia en el área de Botánica, con énfasis en Morfología Vegetal, Anatomía Vegetal, Etnobotánica en el área de Agroecología con énfasis en Valoración y uso de Recursos Fitogenéticos. Se ha desempeñado principalmente en las siguientes temáticas: Amazonía, Frutos Comestibles, Passifloras, Cultivo de Tejidos Vegetales y Sistemas de Información Geográfica (SIG).

\section{Referencias}

[1] Alberto Franco da Silva C, Carlos Bampi A, Silva D, Alberto Franco C. Regional Dynamics of the Brazilian Amazon: between Modernization and Land Conflicts. Revista Colombiana de Geografía 2019; 28:2256-5442. https://doi. org/10.15446/rcdg.v28n2.72872.

[2] Qin Y, Xiao X, Wigneron J-P, Ciais P, Brandt M, Fan L, et al. Carbon loss from forest degradation exceeds that from deforestation in the Brazilian Amazon. Nature Climate Change 2021;11. https://doi.org/10.1038/s41558-021-01026-5.

[3] Araujo LS, Magdalena UR, Louzada TS, Salomon PS, Moraes FC, Ferreira BP, et al. Growing industrialization and poor conservation planning challenge natural resources' management in the Amazon Shelf off Brazil. Marine Policy 2021; 128:104465. https://doi.org/10.1016/J. MARPOL.2021.104465.

[4] Ribeiro SS, Schwartz G, Silva AR, da Cruz DC, Brasil Neto AB, Gama MAP, et al. Soil properties under different supplementary organic fertilizers in a restoration site after kaolin mining in the Eastern Amazon. Ecological Engineering 2021;170:106352. https://doi.org/10.1016/J. ECOLENG.2021.106352.

[5] Swanson AC, Kaplan D, Toh K-B, Marques EE, Bohlman SA. Changes in floodplain hydrology following serial damming of the Tocantins River in the eastern Amazon. Science of The Total Environment 2021;800:149494. https://doi. org/10.1016/J.SCITOTENV.2021.149494.

[6] Moss G, Moss M. Os rios voadores, Um guia didático para explicar aos seus alunos o que são os rios voadores e porque eles têm tudo a ver com a preservação da Amazônia por sua importante contribuição para as chuvas que irrigam gratuitamente nossas plantações e o clima brasileiro. Caderno do professor. 1st ed. Lago Sul, Brasilia, Brasil: Horizonte. Projeto Rios Voadores; 2014.

[7] Jordan L. Fires in Brazil's Pantanal wetland and Amazon rainforest worst in a decade. UnEarthed 2020. https:// unearthed.greenpeace .org/2020/09/04/fires-brazil-pantanalwetland-amazon-rainforest/

[8] World Wide Fund For Nature (WWF). Fires in the Brazilian Pantanal break a record 2020. https://wwf.panda.org/wwf_ news/?602871/Fires-in-the-Brazilian-Pantanal-break-arecord

[9] Guimarães Paiva S, Carvalho Gontijo C, Carvalho Gontijo R, Madrigal L, de Nazaré Klautau-Guimarães M, Fabiana de Oliveira S. Migration in brazilian afro-descendants communities: A new approach to illustrate the meaning of contemporary Quilombo. Revista Da Associação Brasileira de Pesquisador s Negr s - ABPN 2020; 12:188-208. https:// doi.org/10.31418/2177-2770.2020.v12.n.32.p188-208.

[10] Monteiro E, Reis MCG. Patrimônio Afro-Brasileiro no Contexto da Educação Escolar Quilombola. Educação \& Realidade 2019;44:1-22. https://doi.org/10.1590/2175623688369 .

[11] da Silva AL, Begossi A. Biodiversity, food consumption and ecological niche dimension: a study case of the riverine populations from the Rio Negro, Amazonia, Brazil. Environment, Development and Sustainability 2009;11:489_ 507. https://doi.org/10.1007/s10668-007-9126-z.

[12] Instituto Brasileiro de Geografia e Estatística (IBGE). Indicadores IBGE. Estatística da Produção Pecuária. Out.Dez, 2020. Rio de Janeiro, Brasil: 2020. 
[13] Kroeger ME, Meredith LK, Meyer KM, Webster KD, Plinio Barbosa De Camargo, Fonseca De Souza L, et al. Rainforestto-pasture conversion stimulates soil methanogenesis across the Brazilian Amazon. The ISME Journal 2021;15:658-72. https://doi.org/10.1038/s41396-020-00804-x.

[14] Françoso RD, Haidar RF, Machado RB. Tree species of South America central savanna: endemism, marginal areas and the relationship with other biomes. Acta Botanica Brasilica 2016;30. https://doi.org/10.1590/0102-33062015abb0244.

[15] Davis W. El río. Exploraciones y descubrimientos en la Selva Amazónica. 1st ed. Bogotá, Colombia: Banco de la República. El Áncora Editores; 2006.

[16] Global Forest Watch (GFW). How rainforests are formed, and how they are being destroyed. World Resources Institute 2020. https://www.globalforestwatch.org/blog/data-andresearch/tropical-rainforest-ecology-and-threats/

[17] Fondo Mundial para la Naturaleza (WWF). Amazonía Viva Informe 2016: Un enfoque regional para la conservación en la Amazonía. Brasilia, Brasil: 2016.

[18] Ferreira RP, Lopes PFM, Campos-Silva J v., Silvano RAM, Begossi A. The Upper Juruá Extractive Reserve in the Brazilian Amazon: past and present $\dagger$. Brazilian Journal of Biology 2022;82. https://doi.org/10.1590/1519-6984.239188.

[19] Santos AM dos, Silva CFA da, Almeida Junior PM de, Rudke AP, Melo SN de. Deforestation drivers in the Brazilian Amazon: assessing new spatial predictors. Journal of Environmental Management 2021; 294:113020. https://doi. org/10.1016/J.JENVMAN.2021.113020.

[20] Primavesi A. Pergunte ao solo e às raízes: uma análise do solo tropical e mais de 70 casos resolvidos pela agroecologia. vol. 1. 1st ed. São Paulo, Brasil: Nobel; 2014.

[21] Instituto Brasileiro de Geografia e Estatística (IBGE). Legal Amazon 2021. https://www.ibge.gov.br/en/geosciences/ environmental-information/pedology/17927-legal-amazon. $\mathrm{html} ?=\& \mathrm{t}=\mathrm{o}-$ que $-\mathrm{e}$

[22] Bastos RC,Brasil LS, CarvalhoFG, Calvão LB,Silva JO de A,Juen L. Odonata of the state of Maranhão, Brazil: Wallacean shortfall and priority areas for faunistic inventories. Biota Neotropica 2019;19. https://doi.org/10.1590/1676-0611-bn-2019-0734.

[23] Sist P, Piponiot C, Kanashiro M, Pena-Claros M, Putz FE, Schulze M, et al. Sustainability of Brazilian forest concessions. Forest Ecology and Management 2021;496:119440. https:// doi.org/10.1016/J.FORECO.2021.119440.

[24] Canetti A, Braz EM, de Mattos PP, Basso RO, Filho AF. A new approach to maximize the wood production in the sustainable management of Amazon forest. Annals of Forest Science 2021;78. https://doi.org/10.1007/s13595-021-01079-8.
[25] A Baker JC, Garcia-Carreras L, Buermann W, Castilho de Souza D, Marsham JH, Kubota PY, et al. Robust Amazon precipitation projections in climate models that capture realistic land-atmosphere interactions. Environ Res Lett 2021;16:074002. https://doi.org/10.1088/1748-9326/ abfb2e.

[26] Bush MB, Nascimento MN, Åkesson CM, Cárdenes-Sandí GM, Maezumi SY, Behling H, et al. Widespread reforestation before European influence on Amazonia. Science 2021;372. https://doi.org/10.1126/science.abf3870.

[27] Tritsch I, le Tourneau F-M. Population densities and deforestation in the brazilian Amazon: New insights on the current human settlement patterns. Applied Geography 2016;76:163-72. https://doi.org/10.1016/j. apgeog.2016.09.022.

[28] Coelho Prates R, Caetano Bacha CJ. Os processos de desenvolvimento e desmatamento da Amazônia. Economia e Sociedade, Campinas 2011;20:601-36.

[29] Gonçalves E. BR-319: A polêmica pavimentação da rodovia que corta a Amazônia. Veja 2021. https://veja.abril.com.br/ brasil/br-319-a-polemica-pavimentacao-da-rodovia-quecorta-a-amazonia/

[30] Brazilian Agricultural Research Corporation (EMBRAPA). Integrated crop-livestock-forestry systems - ICLFS. Integrated Crop-Livestock-Forestry Systems 2011. https:// www.embrapa.br/en/tema-integracao-lavoura-pecuariafloresta-ilpf/nota-tecnica

[31] Miccolis A, Marson Teixeira de Andrade R, Pacheco P. Landuse trends and environmental governance policies in Brazil. Paths forward for sustainability. Bogor, Indonesia: 2014.

[32] Porro R, Miller RP, Tito MR, Donovan JA, Vivan JL, Trancoso $\mathrm{R}$, et al. Agroforestry in the Amazon region: A pathway for balancing conservation and development, 2012. https://doi. org/10.1007/978-94-007-4676-3_20.

[33] Mustin K, Carvalho WD, Hilário RR, Costa-Neto S v., Silva C, Vasconcelos IM, et al. Biodiversity, threats and conservation challenges in the Cerrado of Amapá, an Amazonian savanna. Nature Conservation 2017;22. https://doi.org/10.3897/ natureconservation. 22.13823

[34] Pajolla M. Nova Transamazônica: reconstrução da BR319 pode reviver tragédia socioambiental. Brasil de Fato 2021. https://www.brasildefato.com.br/2021/03/31/novatransamazonica-reconstrucao-da-br-319-pode-revivertragedia-socioambiental

[35] Conselho Indigenista Missionário (CIMI). Violence against indigenous peoples in Brazil. Data for 2018. Rio de Janeiro, Brasil: 2018. 
[36] Vittor AY, Laporta GZ, Sallum MAM, Walker RT. The COVID-19 crisis and Amazonia's indigenous people: Implications for conservation and global health. World Development 2021;145:105533. https://doi.org/10.1016/J. WORLDDEV.2021.105533.

[37] Rodrigues EPS, Abreu IN, Lima CNC, da Fonseca DLM, Pereira SFG, dos Reis LC, et al. High prevalence of antiSARS-CoV-2 IgG antibody in the Xikrin of Bacajá (Kayapó) indigenous population in the brazilian Amazon. International Journal for Equity in Health 2021;20. https://doi.org/10.1186/ s12939-021-01392-8.

[38] Foschiera A, Silva JS da. A espacialização da Covid-19 nos Distritos Sanitários Especiais Indígenas da Amazônia Legal. Ateliê Geográfico 2020;14. https://doi.org/10.5216/ ag.v14i3.65329.

[39] Programa de Monitoramento da Amazônia e Demais Biomas - PAMZ+, Coordenação-Geral de Observação da Terra CGOBT, Instituto Nacional de Pesquisas Espaciais - INPE. Metodologia Utilizada nos Projetos PRODES e DETER. Sao Pablo, Brasil: 2019.

[40] Montibeller B, Kmoch A, Virro H, Mander Ü, Uuemaa E. Increasing fragmentation of forest cover in Brazil's Legal Amazon from 2001 to 2017. Scientific Reports 2020;10. https://doi.org/10.1038/s41598-020-62591-x.

[41] Brovelli MA, Sun Y, Yordanov V. Monitoring forest change in the Amazon using multi-temporal remote sensing data and machine learning classification on Google Earth engine. ISPRS International Journal of Geo-Information 2020;9. https://doi.org/10.3390/ijgi9100580.

[42] PRODES - Amazônia. Monitoramento do desmatamento da floresta Amazônica brasileira por satélite. Instituto Nacional de Pesquisas Espaciais - INPE 2019. http://www.obt.inpe.br/ OBT/assuntos/programas/amazonia/prodes
[43] Brancalion PHS, Garcia LC, Loyola R, Rodrigues RR, Pillar VD, Lewinsohn TM. Análise crítica da Lei de Proteção da Vegetação Nativa (2012), que substituiu o antigo Código Florestal: atualizações e ações em curso. Natureza \& Conservação 2016;14. https://doi.org/10.1016/j. ncon.2016.03.004.

[44] Presidência da República. Casa Civil. Subchefia para Assuntos Jurídicos. Lei No 12.651, de 25 de maio de 2012. Dispõe sobre a proteção da vegetação nativa; altera as Leis nos 6.938, de 31 de agosto de 1981, 9.393, de 19 de dezembro de 1996, e 11.428, de 22 de dezembro de 2006; revoga as Leis nos 4.771 , de 15 de setembro de 1965 , e 7.754 , de 14 de abril de 1989, e a Medida Provisória no 2.166-67, de 24 de agosto de 2001; e dá outras providências. Brasilia, Brasil: 2012.

[45] Quadros V. Ex-presidente da Funai: Em 42 anos na Amazônia não vi ameaça à soberania. Exame 2019. https://exame.com/ brasil/ex-presidente-da-funai-em-42-anos-na-amazonia-naovi-ameaca-a-soberania/

[46] Veiga J, D'Angelis W. Duas tacadas de Bolsonaro para o extermínio indígena. Outras Palavras Jornalismo de Profundidade e Pos-Capitalismo 2020. https://outraspalavras. net/outrasmidias/duas-tacadas-de-bolsonaro-para-oexterminio-indigena/

[47] Kucharz T. O plano genocida de Bolsonaro para a destruição dos povos indígenas. Instituto Humanista Unisinos (IHU) 2020. http://www.ihu.unisinos.br/78-noticias/596465-oplano-genocida-de-bolsonaro-para-a-destruicao-dos-povosindigenas

[48] Saraiva A. PF troca chefe do órgão no Amazonas após notíciacrime contra Salles. Consultor Jurídico INVESTIGAÇÃO DE MADEIREIROS 2021. https://www.conjur.com.br/2021abr-15/pf-troca-chefe-orgao-amazonas-noticia-crimesalles?atfbqyu $4=548$ 